\title{
Topical nonsteroid anti-inflamatory drugs - indications, efficacy and safety
}

\author{
Diana Mazilu1,2, Ruxandra Ionescu, ${ }^{1,2}$ \\ 1"Carol Davila" University of Medicine, Bucharest, Romania \\ 2"Sf. Maria" Clinical Hospital, Bucharest, Romania
}

\begin{abstract}
Topical Nonsteroid anti-inflammatory drugs are efficient for the treatment of acute and chronic pain in musculo-skeletal diseases. There clinical benefit is similar to the oral form of administration, but with significant less systemic adverse events. There are many forms of topical administration such as gels, cream, spray, patches. The newly topical patch of ibuprofen is an option. The mean level of ibuprofen delivered from the topical patch is consistent with the levels required for therapeutic relief and this dose is much lower that the dose required for oral dosing. Topical nonsteroid anti-inflammatory drugs are a good choice of pain relief for older patients and for patients with cardiovascular and gastrointestinal comorbidities.
\end{abstract}

Keywords: chronic and acute musculoskeletal pain, topical NSAIDs, efficacy and adverse events

\section{INTRODUCTION}

Nonsteroid anti-inflammatory drugs (NSAID) are used for the treatment of acute and chronic pain in inflammatory and degenerative rheumatic diseases, as well as musculo-skeletal soft tissue injuries such as sprains. Their usefulness may be limited due to possible adverse events regarding cardiovascular, gastrointestinal and renal system. The topical formula of NSAID opened the opportunity to obtain NSAID clinical benefits without systemic adverse events. This formula is marketed in Europe for almost 20 years and topical NSAID are part of treatment guidelines in Europe as well as in United Stated for osteoarthritis and pain management.

\section{Efficacy of topical NSAID}

Similarly to oral NSAID, the topical formula induce analgesia, reduces local inflammation by inhibiting cyclooxigenase (COX) and leukotriene systems. Their efficacy depend on the NSAID pharmaceutical properties, product excipients and its absorption, and also the site of application (1). It should be kept in mind the layers of the skin and their properties. The superficial layer, stratum cor- neum is a lipophilic one. The epiderma is predominant aquatic. Beneath these two layers are the basal membrane and the dermum. An efficient topical NSAID should be able to transverse all these layers, to be absorbed and to reach systemic circulation. So, it should be hydrophilic but also hydrophobic (2). The majority of studies that investigated topical NSAIDs efficacy were comparative studies using also the oral form of NSAIDs. The type of NSAID preparations approved nowadays are topical solutions, gel, patch, cream, foam with different compositions and absorption rate, but the mean blood level that is reached is about 5\% (3).

There are several systemic reviews that proved the efficacy of topical NSAID in chronic musculoskeletal pain relief, but also in acute pain musculoskeletal pain, such as in sprains. There is an important relative benefit after topical NSAID administration of 1.7 (95\% CI, 1.5-1.9) in acute conditions and 2.0 (95\% CI, 1.5-2.7) in chronic musculoskeletal diseases (4). Similar results were mentioned in another analysis in patients with acute pain secondary to sprains, strains or sport injuried (5). In another meta-analyses, after 2 weeks of treatment 
the relative benefit was 1.9 (95\% CI, 1.7-2.2) in patients with chronic conditions and the mean treatment response rate was $48 \%$ (1).

The efficacy of topical diclofenac (topical solution, gel, patch, spray) was shown in multiple studies. The majority of them included patients with knee osteoarthritis. It is clear now that topical administration of diclofenac is similar to oral administration regarding knee pain reduction (6). But comparing these two types of administration, the topical form does not interfere with plachetar aggregation and it inhibits in a lesser degree the activity of COX1 and COX-2. After topical administration the systemic exposure is 5 to 7 times lower than the oral exposure of diclofenac (7).

There are extensive clinical trials that showed ibuprofen efficacy in different local formulations such as cream, gel, spray and patch. The first studies included patients with osteoarthritis. Using gel formula, the relative bioavailability of ibuprofen after administration of $500 \mathrm{mg}$ in $5 \%$ gel was $22 \%$, with the time to peak concentrations was about 2.4 hours (8). Regarding topical patch, the mean level of ibuprofen delivered from the patch was $32.2 \mathrm{mg}$ per day during a 5-day period. The systemic absorption of ibuprofen from a 200-mg patch is low and the levels of ibuprofen leaving the patch during a 24-hour exposure are consistent with the levels required for therapeutic relief. Pain relief with ibuprofen patch is achieved at doses much lower than those required for oral dosing (9).

Similar results were obtained for ketoprofen (3). As much as $63 \%$ of the patients benefit form topical ketoprofen. The majority of the studies included patients with knee osteoarthritis. Other NSAID approved for topical usage are felbinac, piroxicam, nimesulide, etoricoxib (3).

\section{Safety and tolerability of topical NSAID}

The available evidence suggests that currently available formulations of topical NSAIDs have generally similar safety profile. The majority of the studies that evaluated topical NSAIDs efficacy compared this form of administration to the oral form. Their efficacy is similar, but the incidence of adverse events is significantly lower and this is very important in clinical practice. The overall results suggest that topical administration of NSAIDs has a good safety profile $(10,11)$. Topical NSAID are associated with lower risk of gastrointestinal complications such as bleeding or perforation (12-14), renal toxicity (13), cardiovascular adverse events $(4,5,13)$. The most frequent adverse events reported were related to skin reactions (3). In studies comparing topical diclofenac versus placebo, local skin dryness and skin irritation were frequently found (6). When comparing the oral form of administration versus the topical one, there were cases reported with increase of liver enzymes, creatinine and decrease in the level of hemoglobin in patients with oral form of administration (6).

Many topical formulations contain dimethyl sulfoxide (DMSO) as a penetration enhancer. DMSO may be the cause of some adverse events such as minor erythema, pruritus and burning as a result of possible vasodilation. They are reversible after treatment discontinuation (1). DMSO may induce hypersensitivity reactions due to histamine release, neuropathy and chest pain (1). It seems that the highest incidence for contact allergy is with topical ketoprofen when it was compared to other NSAID such as naproxen, piroxicam, diclofenac and others (15). Also, topical ketoprofen is associated with photoallergy (10). The newly approved ibuprofen in the form of plaster was shown to be well tolerated with low irritancy (16).

\section{Clinical utility}

Hand osteoarthritis is a very common musculoskeletal disease with high prevalence with increasing age. In the newly published 2018 EULAR recommendations regarding treatment of hand osteoarthritis, the topical treatments are preferred over systemic treatments because of safety reasons. Topical NSAIDs are the first pharmacological topical treatment of choice as they show similar pain relief as oral NSAIDs (17).

The recommendations for the management of large joints, such as knee and hip, osteoarthritis were published by ACR in 2012 (18). It is clearly stated that persons age $\geq 75$ years with knee osteoarthritis should use topical rather than oral NSAIDs (18). These recommendations are based on the evidence regarding benefit, safety/tolerability and the consensus judgments of clinical experts.

Beside osteoarthritis, topical NSAIDs are used for acute injuries such as sport injuries (sprains, strains, contusions), but the use of topical NSAIDs is not appropriate in patients with skin injuries, allergies to the active drug formula, or excipients such as 
penetration enhancer. One of the first reviews on topical NSAIDs concluded that this form of administration is more effective than placebo, and comparable to oral NSAIDs in analgesic efficacy (4).

\section{CONCLUSION}

Topical NSAIDs in one of the cornerstone for the treatment of chronic and acute musculoskeletal pain. The published data suggests that topical NSAIDs produce effective drug concentrations in dermis,

\section{REFERENCES}

1. Barkin RL. Topical Nonsteroidal Anti-Inflammatory Drugs. Am J Ther 2015;22(5):388-407.

2. Galer BS. All topical NSAIDs not created equal - Understanding topical analgesic drug formulations. Pain 2008;139(1):237-8.

3. Derry S, Conaghan P, Da Silva JAP, Wiffen PJ, Moore RA. Topical NSAIDs for chronic musculoskeletal pain in adults. Cochrane database Syst Rev 2016;4:CD007400.

4. Moore RA, Tramèr MR, Carroll D, Wiffen PJ, McQuay HJ. Quantitative systematic review of topically applied non-steroidal anti-inflammatory drugs. BMJ 1998;316(7128):333-8.

5. Mason L, Moore RA, Edwards JE, Derry S, McQuay HJ. Topical NSAIDs for chronic musculoskeletal pain: systematic review and meta-analysis. BMC Musculoskelet Disord 2004;5(1):28.

6. Roth SH, Fuller P. Diclofenac topical solution compared with oral diclofenac: a pooled safety analysis. J Pain Res 2011;4:159-67.

7. Kienzler J-L, Gold M, Nollevaux F. Systemic bioavailability of topical diclofenac sodium gel $1 \%$ versus oral diclofenac sodium in healthy volunteers. J Clin Pharmacol 2010;50(1):50-61.

8. Kleinbloesem $\mathrm{CH}$, Ouwerkerk M, Spitznagel W, Wilkinson FE, Kaiser RR. Pharmacokinetics and bioavailability of percutaneous ibuprofen. Arzneimittelforschung 1995;45(10):1117-21.

9. Lewis F, Connolly MP, Bhatt A. A Pharmacokinetic Study of an Ibuprofen Topical Patch in Healthy Male and Female Adult Volunteers. Clin Pharmacol Drug Dev 2018;7(7):684-91.

10. Zacher J, Altman R, Bellamy N, et al. Topical diclofenac and its role in pain and inflammation: an evidence-based review. Curr Med Res Opin 2008;24(4):925-50. muscle, synovium and in the joints proximal to and below the application site (14). The ibuprofen patch applied during a 5-day period can result in effective pain relief at doses much lower than those required for oral dosing $-2.7 \%(32.2 / 1,200 \mathrm{mg})$ of the potential daily maximum dose. Ibuprofen patch is an option for acute or chronic musculoskeletal pain due to its rapidly absorption, easy application, low dose efficacy and unnecessary systemic exposure.

\section{Conflict of interest: none declared Financial support: none declared}

11. Taylor RS, Fotopoulos G, Maibach H. Safety profile of topical diclofenac: a meta-analysis of blinded, randomized, controlled trials in musculoskeletal conditions. Curr Med Res Opin 2011;27(3):60522.

12. Evans JM, McMahon AD, McGilchrist MM, et al. Topical nonsteroidal anti-inflammatory drugs and admission to hospital for upper gastrointestinal bleeding and perforation: a record linkage case-control study. BMJ 1995;311(6996):22-6.

13. Altman R, Barkin RL. Topical Therapy for Osteoarthritis: Clinical and Pharmacologic Perspectives. Postgrad Med 2009;121(2):13947.

14. Haroutiunian S, Drennan DA, Lipman AG. Topical NSAID therapy for musculoskeletal pain. Pain Med 2010;11(4):535-49.

15. Diaz RL, Gardeazabal J, Manrique P, et al. Greater allergenicity of topical ketoprofen in contact dermatitis confirmed by use. Contact Dermatitis 2006;54(5):239-43.

16. Maganji M, Connolly MP, Bhatt A. Cutaneous irritancy of an ibuprofen medicated plaster in healthy volunteers. Postgrad Med 2018;130(1):19-23.

17. Kloppenburg M, Kroon FP, Blanco FJ, et al. 2018 update of the EULAR recommendations for the management of hand osteoarthritis. Ann Rheum Dis 2019;78(1):16-24.

18. Hochberg MC, Altman RD, April KT, et al. American College of Rheumatology 2012 recommendations for the use of nonpharmacologic and pharmacologic therapies in osteoarthritis of the hand, hip, and knee. Arthritis Care Res (Hoboken) 2012;64(4):465-74. 